\title{
Article \\ ZnCDs/ZnO@ZIF-8 Zeolite Composites for the Photocatalytic Degradation of Tetracycline
}

\author{
Yong Cheng *, Xiuxiu Wang, Yu Mei, Dan Wang and Changchun Ji
}

The Key Laboratory of Functional Molecular Solids, Ministry of Education, Anhui Laboratory of Molecule-Based Materials (State Key Laboratory Cultivation Base), College of Chemistry and Materials Science, Anhui Normal University, Wuhu 241002, China; ahnuwxx@163.com (X.W.); 18815799910@ahnu.edu.cn (Y.M.); ahnudw@163.com (D.W.); lovebama@ahnu.edu.cn (C.J.)

* Correspondence: chyong2008happy@163.com

\begin{abstract}
Considering the photocatalytic performance of $\mathrm{CDs}, \mathrm{ZnO}$, and the unique porous nanostructure and stability of ZIF-8, we prepared ZnCDs/ZnO@ZIF-8 zeolite composites. The resultant material represented an enhanced ability for the photodegradation of TC compared with that of $\mathrm{ZnCDs}$ and $\mathrm{ZnO}$. The photocatalytic degradation efficiency reached over $85 \%$. The catalytic activity of the composites was maintained after four cycles. The experimental result indicated that $\cdot \mathrm{O}_{2}$ radical was the active species in the reaction.
\end{abstract}

Keywords: carbon dots; zeolite composites; photocatalytic; tetracycline

Citation: Cheng, Y.; Wang, X.;

Mei, Y.; Wang, D.; Ji, C.

ZnCDs/ZnO@ZIF-8 Zeolite

Composites for the Photocatalytic

Degradation of Tetracycline. Catalysts

2021, 11, 934. https://doi.org/

$10.3390 /$ catal11080934

Academic Editor: Andres Aguayo

Received: 13 May 2021

Accepted: 20 July 2021

Published: 30 July 2021

Publisher's Note: MDPI stays neutra with regard to jurisdictional claims in published maps and institutional affiliations.

Copyright: (c) 2021 by the authors. Licensee MDPI, Basel, Switzerland. This article is an open access article distributed under the terms and conditions of the Creative Commons Attribution (CC BY) license (https:// creativecommons.org/licenses/by/ $4.0 /)$

\section{Introduction}

Tetracycline (TC) is widely used as a broad-spectrum antibiotic for treating a wide range of diseases, such as mycoplasmal pneumonia, hydatoncus, and the diagnosis of malignant tumor epidemic typhus [1,2]. However, as with other antibiotics, tetracycline can be only partially metabolized or absorbed by humans and animals and its residue ends up in wastewater [3]. Tetracycline residue in hydrosphere inevitably brings underlying hazard to human health. Consequently, the removal of TC from aqueous solutions is a crucial and significant goal. So far, researchers have explored various methods such as adsorption [4], biodegradation [5,6], and electrochemical [7] to combat pollution by antibiotics. However, most methods have been restricted owing to lack of efficiency, costliness, or sophisticated instrumentation. Photocatalytic technology has gained attention for the degradation of TC owing to its high efficiency, low cost, and environmentally friendly properties [8-10].

MOF-based composites have attracted much attention in recent years for their potential uses in adsorption [11], photocatalysis [12,13], fluorescence sensing [14], etc. Among of them, zeolitic imidazolate framework (ZIF-8), which is comprised of zinc and 2-methyl imidazole (2-mIm), is considered a highly appropriate host matrix to form composites owing to its exceptional chemical and thermal stability and relatively large cavities $(11.6 \sim 3.8 \AA)[15,16]$. Various materials have been successfully combined with ZIF-8 and have acquired a synergistic effect [17-20]. For example, $\mathrm{ZnO}$ is a semiconductor and has been widely used as a photocatalyst, but it remains challenging to capture the target molecules for enhanced photocatalysis. It has been found that higher photocatalytic ability and photochemical activities were achieved as $\mathrm{ZnO}$ hybridized with ZIF-8 [21-23]. Nevertheless, the charge conductivity of ZnO@ZIF-8 is still unsatisfactory, which limits the improvement of photocatalytic capacity further.

Carbon dots (CDs)-based materials have been studied as a new generation of photocatalytic composites owing to the tunable photoluminescence and high efficiency light capturing capability [24-26]. For example, $\mathrm{CDs} @ \mathrm{TiO}_{2}$ absorbs more visible light compared to bare $\mathrm{TiO}_{2}$, which helps to increase the catalytic efficiency [27]. CDs@ZnO produced a newly formed conduction band, which contributes to an increased level of electron 
migration to the valence band and thereby enhances their photocatalytic activity $[28,29]$. It is proposed that CDs can modulate the band structure of semiconductors and improve the optical properties and impart novel functionalities to the as-synthesized composites. So, it is anticipated that higher photocatalysis should be achieved by incorporating CDs into ZnO@ZIF-8.

Herein, we prepared a ZnCDs/ZnO@ZIF-8 zeolite composite, in which $\mathrm{ZnCDs/ZnO}$ heterojunction was encapsulated by ZIF-8 crystal and formed a core-shell structure. It indicated that the resulting hybrid material exhibits excellent photocatalytic performance for the degradation of tetracycline. The photocatalytic efficiency reached over $85 \%$ after four cycles. The possible reaction mechanism was investigated using the radical scavenger technique. It is hoped that this work could pave the way for developing more photocatalytic zeolite composites.

\section{Results and Discussion}

\subsection{Synthesis of ZnCDs/ZnO@ZIF-8}

The synthesis procedure of ZnCDs/ZnO@ZIF-8 composites is shown in Scheme 1. The mixture of zinc nitrate hexahydrate, folic acid, and DMF was heated at $190{ }^{\circ} \mathrm{C}$, and then cooled down. The product was separated into two parts: the solution was dialyzed and dried to obtain ZnCDs; the residual precipitate was calcined to form $\mathrm{ZnO}$ nanosphere, which was used as template for the preparation of $\mathrm{ZnCDs} / \mathrm{ZnO}$ heterostructures. Finally, 2-methylimidazole was coordinated with zinc ions in the suspension of $\mathrm{ZnCDs} / \mathrm{ZnO}$ to form the ZIF-8 crystal, which wrapped around $\mathrm{ZnCDs} / \mathrm{ZnO}$ heterojunction nanosphere.

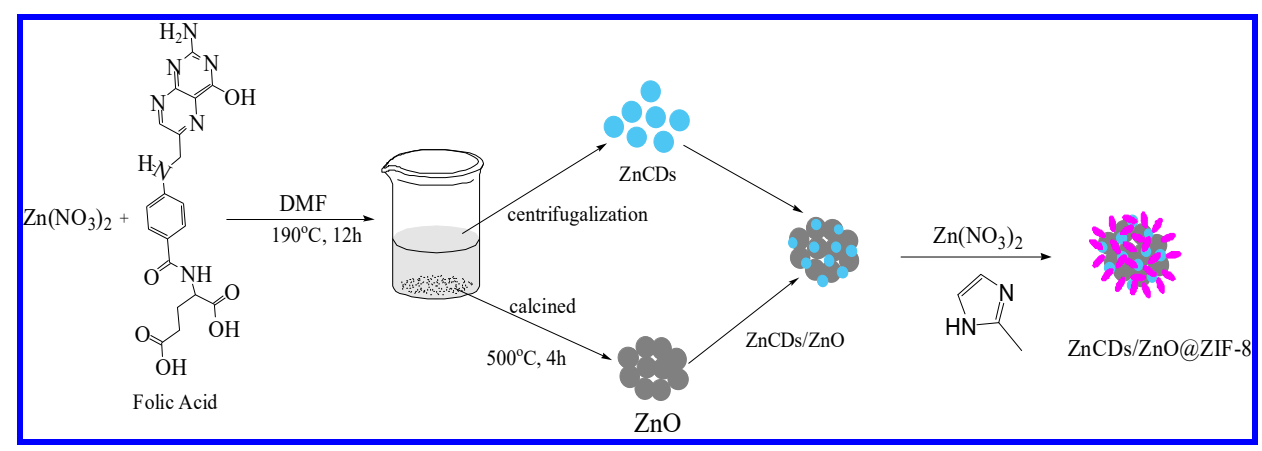

Scheme 1. Synthesis of ZnCDs/ZnO@ZIF-8 composites.

\subsection{Characterization of ZnCDs/ZnO@ZIF-8}

SEM imaging indicates that the morphology of $\mathrm{ZnCDs} / \mathrm{ZnO}$ heterojunction was spherical in shape with a uniform size about $500 \mathrm{~nm}$ (Figure 1a). The ZnCDs/ZnO nanosphere was rough owing to aggregation of nanoparticles. Further observation from Figure $1 \mathrm{~b}$ shows that many polyhedrons of ZIF-8 ( 50 nm) appeared on the surface of ZnCDs/ZnO. Energy-dispersive X-ray spectroscopy (EDX) was performed on the as-prepared composites which determined the elemental composition to comprise $\mathrm{Zn}(26.35), \mathrm{N}$ (3.07), O (34.80), C (35.78) wt.\%.

TEM analysis of ZnCDs/ZnO@ZIF-8 composites suggests a core-shell nanosphere, where the inner core is $\mathrm{ZnCDs} / \mathrm{ZnO}$ heterojunction (Figure 1c) and the outer shell is $\mathrm{ZIF}-8$ crystal (Figure 1d). Figure $2 \mathrm{a}-\mathrm{d}$ are the elemental mapping diagrams of $\mathrm{Zn}, \mathrm{O}, \mathrm{C}$, and $\mathrm{N}$ in $\mathrm{ZnCDs} / \mathrm{ZnO} @ \mathrm{ZIF}-8$ composites, respectively. It can be seen from the figures that these elements were evenly distributed on the materials. 


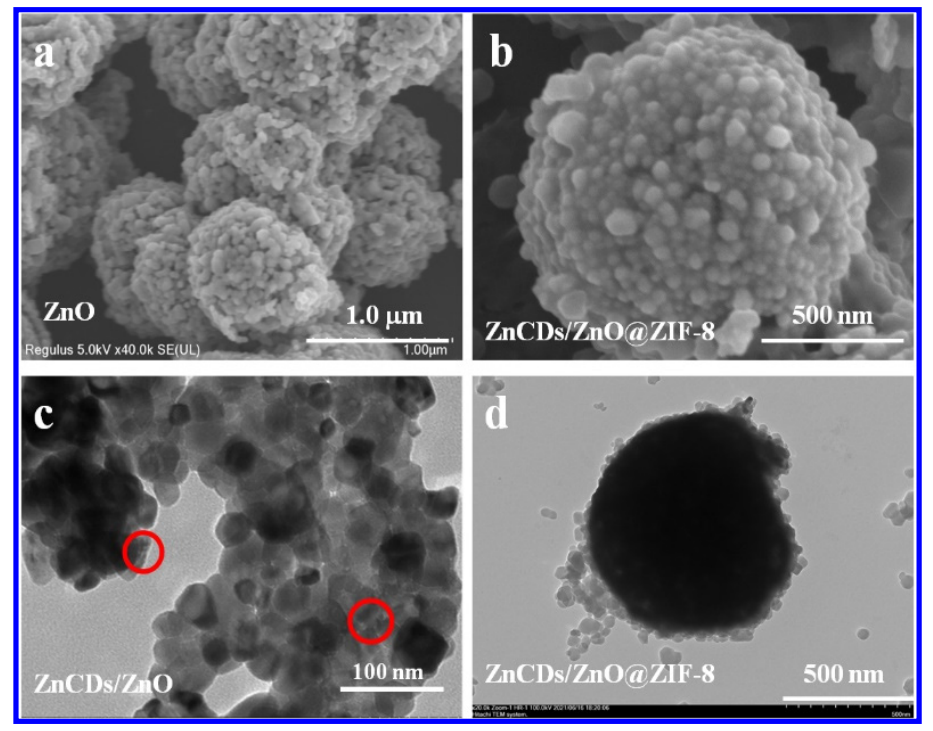

Figure 1. (a) SEM of ZnO (b) SEM of ZnCDs/ZnO@ZIF-8 (c) TEM of ZnCDs/ZnO (d) TEM of ZnCDs/ZnO@ZIF-8 composites.

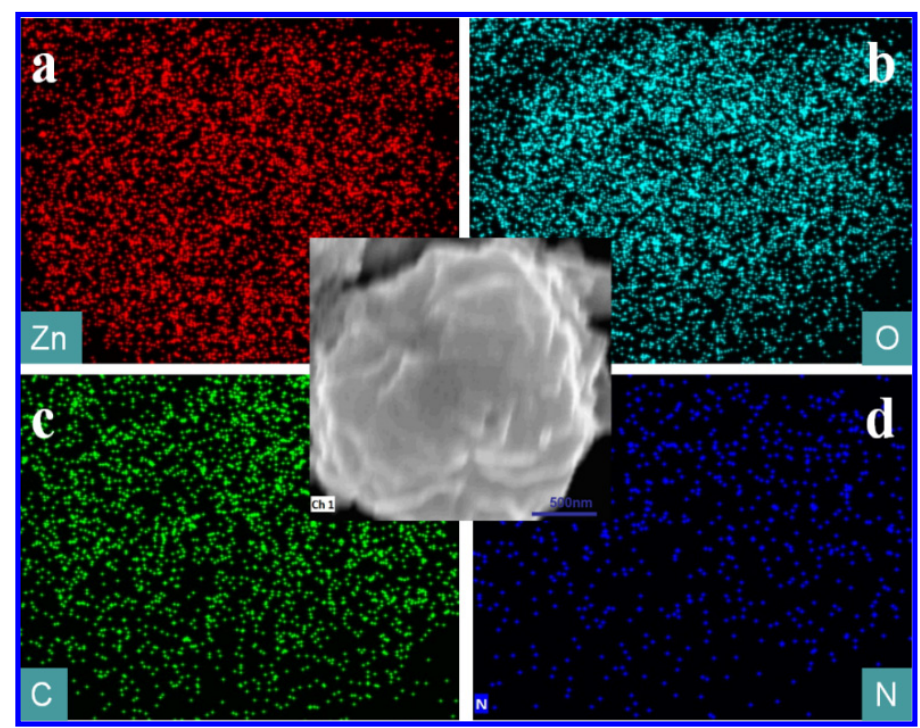

Figure 2. Mapping of ZnCDs/ZnO@ZIF-8 composites (a) Zn (b) O (c) C (d) N.

XRD analysis of ZnCDs/ZnO@ZIF-8 shows a series of typical characteristic peaks with $2 \theta$ at $7.32^{\circ}(39.0), 10.36^{\circ}(41.7), 12.71^{\circ}(44.8), 14.68^{\circ}(56.5), 16.42^{\circ}(40.5), 18.01^{\circ}(41.4)$, and $22.09^{\circ}(54.5)$, because of the (011), (002), (112), (022), (013), and (222), (114) diffraction of ZIF-8 [30]; and the obvious diffraction peaks at $31.77^{\circ}(2.81), 34.43^{\circ}(2.60), 36.26^{\circ}(2.47)$, $47.55^{\circ}(1.91), 56.61^{\circ}(1.62), 62.87^{\circ}(1.47)$, and $67.96^{\circ}(1.37)$, respectively, correspond to the (100), (002), (101), (102), (110), (103), and (112) of the tetragonal phase ZnO (JCPDS, No. 890511) [31] (Figure 3a). The interplanar distances are listed in the parentheses, respectively. It shows that ZnCDs/ZnO@ZIF-8 composites were successfully synthesized. 

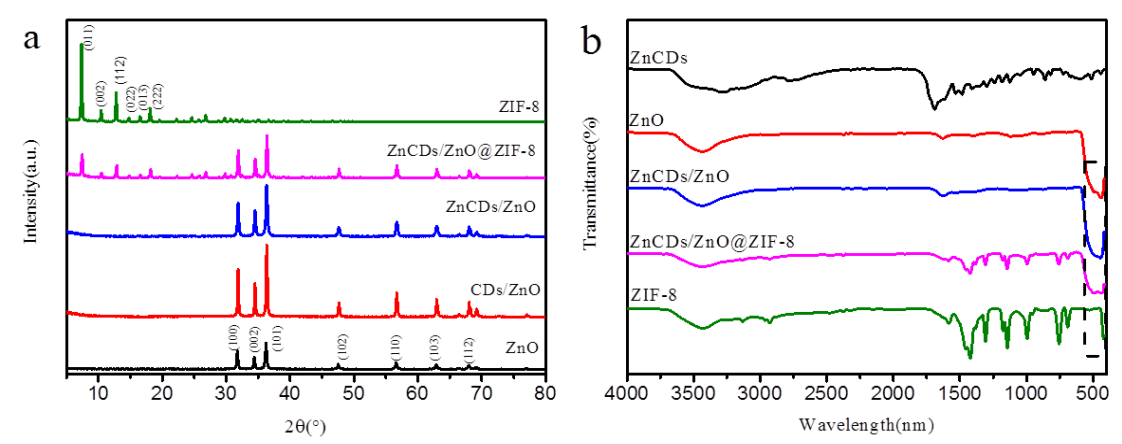

Figure 3. (a) XRD and (b) IR of ZnCDs/ZnO@ZIF-8 composites and related materials.

The FT-IR spectra also confirm the successful synthesis of the composites (Figure 3b). The ZIF-8 and ZnCDs/ZnO@ZIF-8 both exhibits adsorption peaks at 424, 752, 1141, 1306, $1423,1582,2928$, and $3143 \mathrm{~cm}^{-1}$, The adsorption peaks at $1582 \mathrm{~cm}^{-1}$ is attributed to the stretching vibration of $\mathrm{C}=\mathrm{N}$ bonds. The adsorption peaks at 752,1141 , and $1306 \mathrm{~cm}^{-1}$ are attributed to the bending vibrations of the imidazole ring, while the one at $1423 \mathrm{~cm}^{-1}$ is attributed to the stretching vibration of the imidazole ring [21,32]. The adsorption peaks at $2928 \mathrm{~cm}^{-1}$ and $3143 \mathrm{~cm}^{-1}$ are attributed to the aromatic and aliphatic $\mathrm{C}-\mathrm{H}$ stretches [33]. The widened vibration peak between $400 \sim 500 \mathrm{~cm}^{-1}$ in $\mathrm{ZnCDs} / \mathrm{ZnO} @ Z I F-$ 8 could be attributed to the $\mathrm{Zn}-\mathrm{O}$ stretch in $\mathrm{ZnO}$ [28], which indicates $\mathrm{ZnCDs} / \mathrm{ZnO}^{\prime} \mathrm{s}$ successful modification with ZIF-8. 


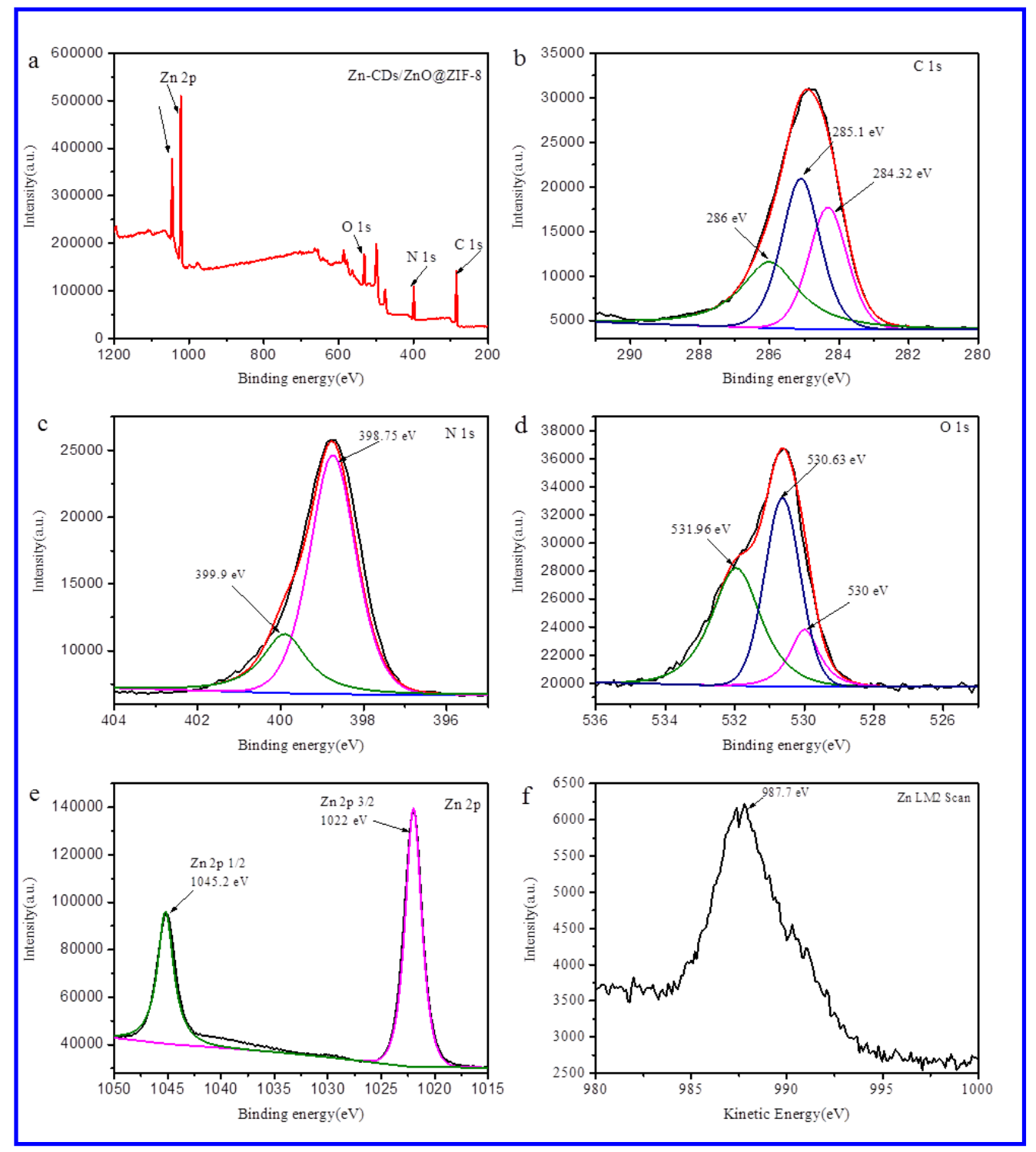

Figure 4. (a) Survey scan (b) C $1 \mathrm{~s}$ (c) N1 s (d) O 1 s (e) Zn2p of XPS and (f) Zn LM2 Scan Auger peak of ZnCDs/ZnO@ZIF-8.

XPS analysis was carried out to understand the surface composition and electronic states of the constituent elements present in the composites. In common, the high-resolution survey scan of ZnCDs/ZnO@ZIF-8 revealed four peaks of Zn(2p), C (1 s), N (1 s), and O (1 s) at the ranges of 1045.2, 285.1, 398.7, $530.6 \mathrm{eV}$, respectively (Figure $4 \mathrm{a})$. The $\mathrm{C}(1 \mathrm{~s})$ spectrum further splits into three peaks at binding energies of 284.32, 285.1, and $286.0 \mathrm{eV}$, which are attributed to $\mathrm{C}=\mathrm{C} / \mathrm{C}-\mathrm{C}, \mathrm{C}-\mathrm{N} / \mathrm{O}, \mathrm{C}=\mathrm{N}$, respectively [34] (Figure $4 \mathrm{~b}$ ). The presence of $\mathrm{Zn}-\mathrm{O}$ and hydroxyl group is determined from the XPS spectrum of $\mathrm{O} 1 \mathrm{~s}$. The peaks are centered at 530.0, 530.63, and $531.96 \mathrm{eV}$, respectively (Figure 4c). All of the peak positions are aligned with the literature [35]. The high-resolution spectrum of $\mathrm{N}(1 \mathrm{~s})$ revealed a dominant peak at $398.75 \mathrm{eV}$, which deconvoluted into two peaks at $398.75 \mathrm{eV}$ and $399.9 \mathrm{eV}$, and corresponded to $\mathrm{N}-\mathrm{C}$ or $\mathrm{N}-\mathrm{H}$, respectively [36] (Figure 4d). The peaks of $\mathrm{Zn} \mathrm{2p}$ at $1022.0 \mathrm{eV}$ and $1045.2 \mathrm{eV}$ assigned to $\mathrm{Zn} 2 \mathrm{p}_{3 / 2}$ and $\mathrm{Zn} 2 \mathrm{p}_{1 / 2}$, respectively (Figure 4e), proved the presence of $\mathrm{Zn}^{2+}$ in the sample [29]. The high-resolution XPS spectra of bare $\mathrm{ZnO}$ indicates the peaks at 1021.8 and $1044.9 \mathrm{eV}$, which were reported earlier [37]. Zn LM2 Scan Auger peak of ZnCDs/ZnO@ZIF-8, as shown in Figure 4f, also indicated that the formation of the composites [38]. 


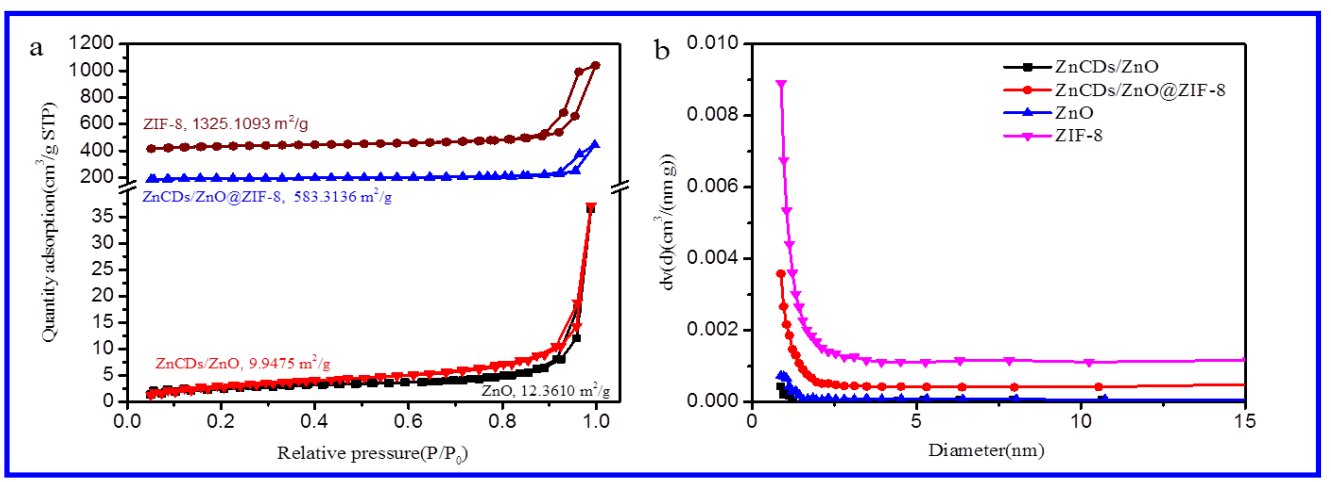

Figure 5. (a) Nitrogen adsorption-desorption isotherm (b) BET of ZnCDs/ZnO@ZIF-8.

Figure 5a shows the nitrogen adsorption-desorption isotherms of ZnCDs/ZnO@ZIF8 and related materials $\mathrm{ZnO}, \mathrm{ZnCDs} / \mathrm{ZnO}$, and ZIF-8. The curves of ZIF-8 are a typical type I nitrogen adsorption-desorption isotherm [39], which fit well with the microporous frameworks of ZIF-8. The curves for ZnCDs/ZnO are a type IV isotherm [39], which shows low adsorption at low pressure and multilayer adsorption at high pressures owing to slits caused by assembly of $\mathrm{ZnO}$. The specific BET surface area is $9.94 \mathrm{~m}^{2} / \mathrm{g}$. For comparison, the BET surface area of ZnCDs/ZnO@ZIF-8 is $583.3 \mathrm{~m}^{2} / \mathrm{g}$, higher than that of ZnCDs/ZnO. The initial adsorption of ZnCDs/ZnO@ZIF-8 is higher than that of $\mathrm{ZnO}$, indicating that micropores exist in ZnCDs/ZnO@ZIF-8, which can be attributed to the frameworks of ZIF-8. The multilayer adsorption at high pressure corresponds to spherical morphology of the sample, similar to that of $\mathrm{ZnO}$ [21]. Figure $5 \mathrm{~b}$ summarized pore size distribution by $\mathrm{BJH}$ method [40]. It indicates that the pore size of ZnCDs/ZnO@ZIF-8 distribution is wide.
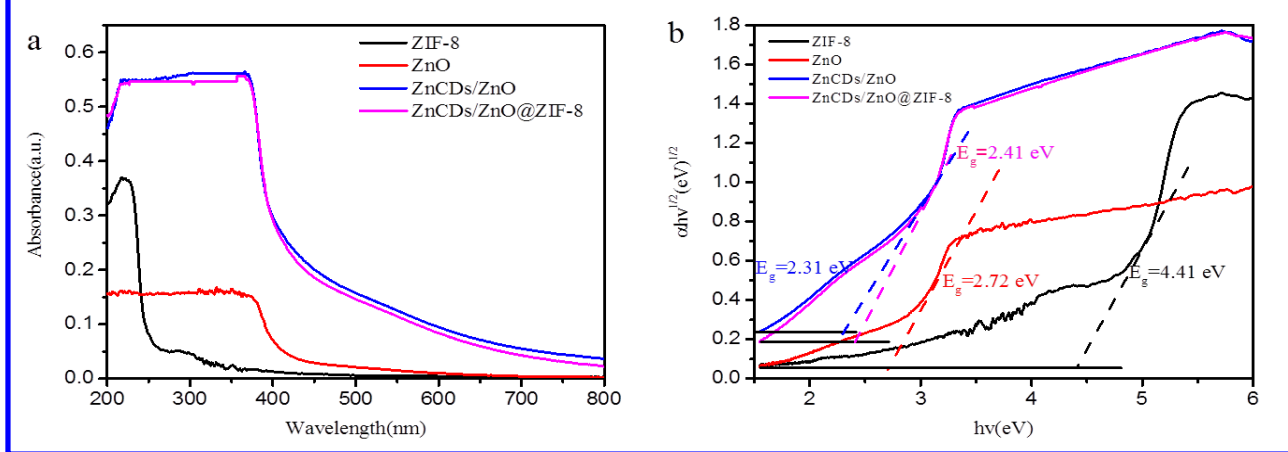

Figure 6. (a) UV-vis DRS (b) Kubelka-Munk function spectrum.

The optical absorption properties of ZnCDs/ZnO@ZIF-8 and various related materials are shown in Figure 6a. All the absorptions occurred in the wavelength range of $200 \sim 400 \mathrm{~nm}$. The observation indicates that the absorption intensity of ZnCDs/ZnO@ZIF-8 is higher than that of $\mathrm{ZnO}$ nanospheres and ZIF-8, respectively. The absorption ability in the visible-light region is enhanced as $\mathrm{ZnCDs} / \mathrm{ZnO}$ enveloped by $\mathrm{ZIF}-8$, which is conducive to the utilization of the solar spectrum. The band gap energies (E) of direct transition semiconductor can be calculated according to the plot of $(\mathrm{ahv})^{2}$ versus photon energy (hv) [41]. As shown in Figure 6b, the $\mathrm{E}_{\mathrm{g}}$ of ZnCDs/ZnO@ZIF-8 estimated to be $2.41 \mathrm{eV}$, which is narrower than that of $\mathrm{ZnO}(2.72 \mathrm{eV})$ and ZIF-8 $(4.41 \mathrm{eV})$, respectively, owing to the incorporation of $\mathrm{ZnCDs} / \mathrm{ZnO}$ with $\mathrm{ZIF}-8$. 


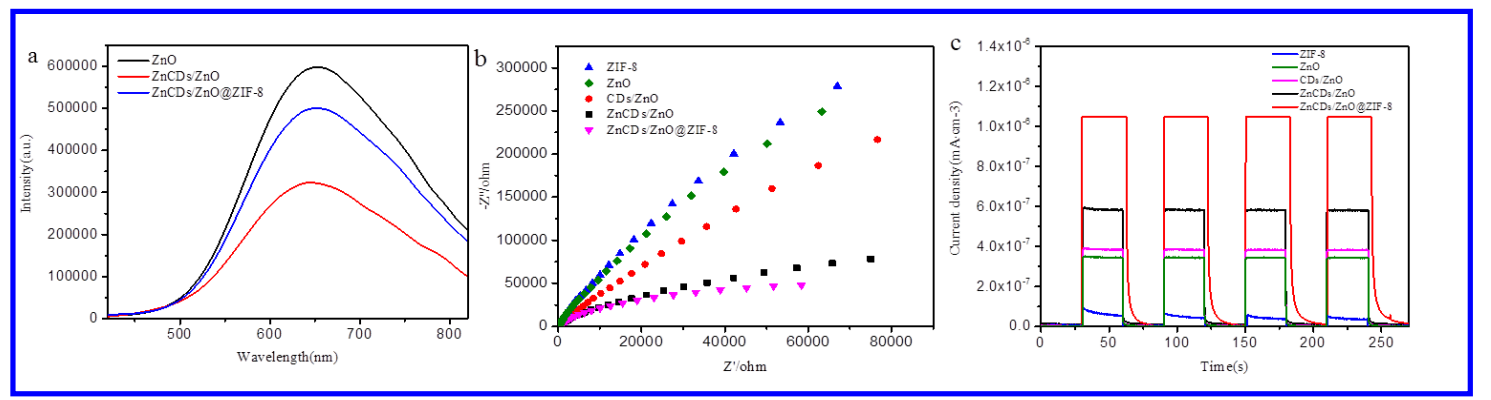

Figure 7. (a) Fluorescence emission. (b) Electrochemical impedance spectrum. (c) Transient photocurrent response of ZnCDs/ZnO@ZIF-8 and related materials.

The fluorescence spectra of the as-synthesized composites and the related samples exhibit emission peaks centered at around $650 \mathrm{~nm}$ (Figure 7a). The absorbance intensity of ZnCDs/ZnO@ZIF-8 increased with respect to that of ZnCDs/ZnO and ZIF-8. As everyone knows, the radius of each arc is relevant to charge-transfer resistance: the smaller the radius of the arc, the lower the corresponding impedance [42]. As displayed in Figure $7 \mathrm{~b}$, ZnCDs/ZnO@ZIF-8 shows the smallest charge-transfer resistance compared with that of $\mathrm{ZnCDs} / \mathrm{ZnO}, \mathrm{ZnO}$, and $\mathrm{ZIF}-8$, coinciding with the conclusion of the EIS results. The transient photocurrent responses and electrochemical impedance spectra of the composites were employed to further explore the separation efficiency of the photogenerated charge carriers [43]. As shown in Figure 7c, the photocurrent responses of ZIF-8 are quite low, which may stem from the fast recombination of electron-hole pairs. Compared with $\mathrm{ZnCDs} / \mathrm{ZnO}, \mathrm{ZnO}$, and ZIF-8, ZnCDs/ZnO@ZIF-8 composites exhibits the highest photocurrent density. It can generate considerably enhanced photo-currents with four visible-light irradiation on-off cycles. This indicates $\mathrm{ZnCDs} / \mathrm{ZnO}$-loading accelerates the transfer of the photo-generated electrons and holes at the interface between $\mathrm{ZnCDs} / \mathrm{ZnO}$ and ZIF-8.

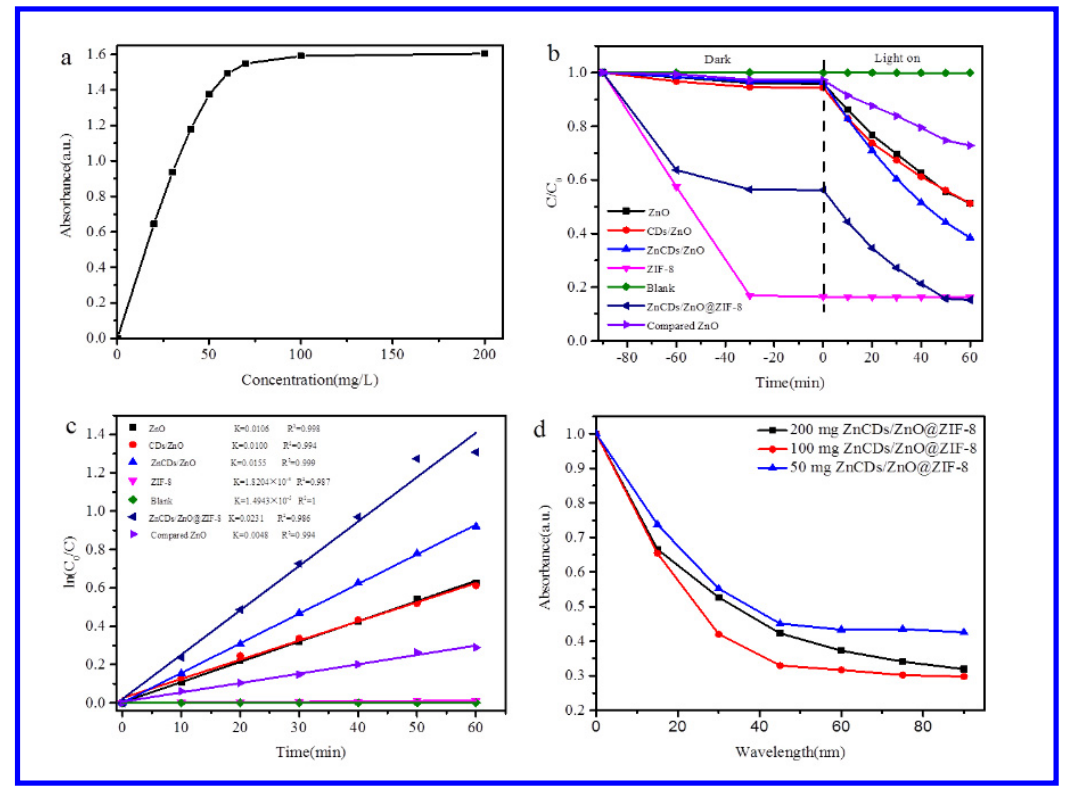

Figure 8. (a) Linear relationship between the concentration and Uv-vis adsorbance of TC. (b) The photocatalytic performance of verious materials for the degradation of TC. (c) Linear relationship of degradation kinetics over ZnCDs/ZnO@ZIF-8 and other related materials. (d) The photocatalytic activity with different qualities of ZnCDs/ZnO@ZIF-8. 


\subsection{Catalytic Performance of ZnCDs/ZnO@ZIF-8}

Photocatalysis is one of the environmentally friendly and sustainable strategies to eliminate the TC pollution [44]. Here, ZnCDs/ZnO@ZIF-8 was evaluated for the photocatalytic degradation of TC under visible-light illumination $(\lambda>420 \mathrm{~nm})$. Figure $8 \mathrm{a}$ is the linear relationship between the concentration and Uv-vis adsorbance of TC. Before the photocatalysis experiments, the suspension was magnetically stirred in the dark for $60 \mathrm{~min}$ to make sure an adsorption-desorption equilibrium containing the catalyst powders and TC aqueous without illumination. As shown in Figure 8b, the photolysis of TC in the absence of photocatalysts can be neglected, which demonstrates that TC is very stable and hardly decomposed. Apparently, there was little adsorption phenomenon in $\mathrm{ZnO}$, $\mathrm{ZnCDs} / \mathrm{ZnO}$, and $\mathrm{CDs} / \mathrm{ZnO}$, while the samples decorated with ZIF-8 showed a discernible adsorption. This phenomenon could be ascribed to the strong adsorption capacity of the wide nanopores interconnected through $0.34 \mathrm{~nm}$ windows assembled as an analogue zeolite sodalite structure of ZIF-8 [45,46]. Significant degradation of TC occurs when ZnCDs/ZnO hybridized with ZIF-8 (Figure 8c). Furthermore, the degradation rate was effect by the quantity of ZnCDs/ZnO@ZIF-8, as depicted in Figure 8d, the optimum dosage is $100 \mathrm{mg}$.

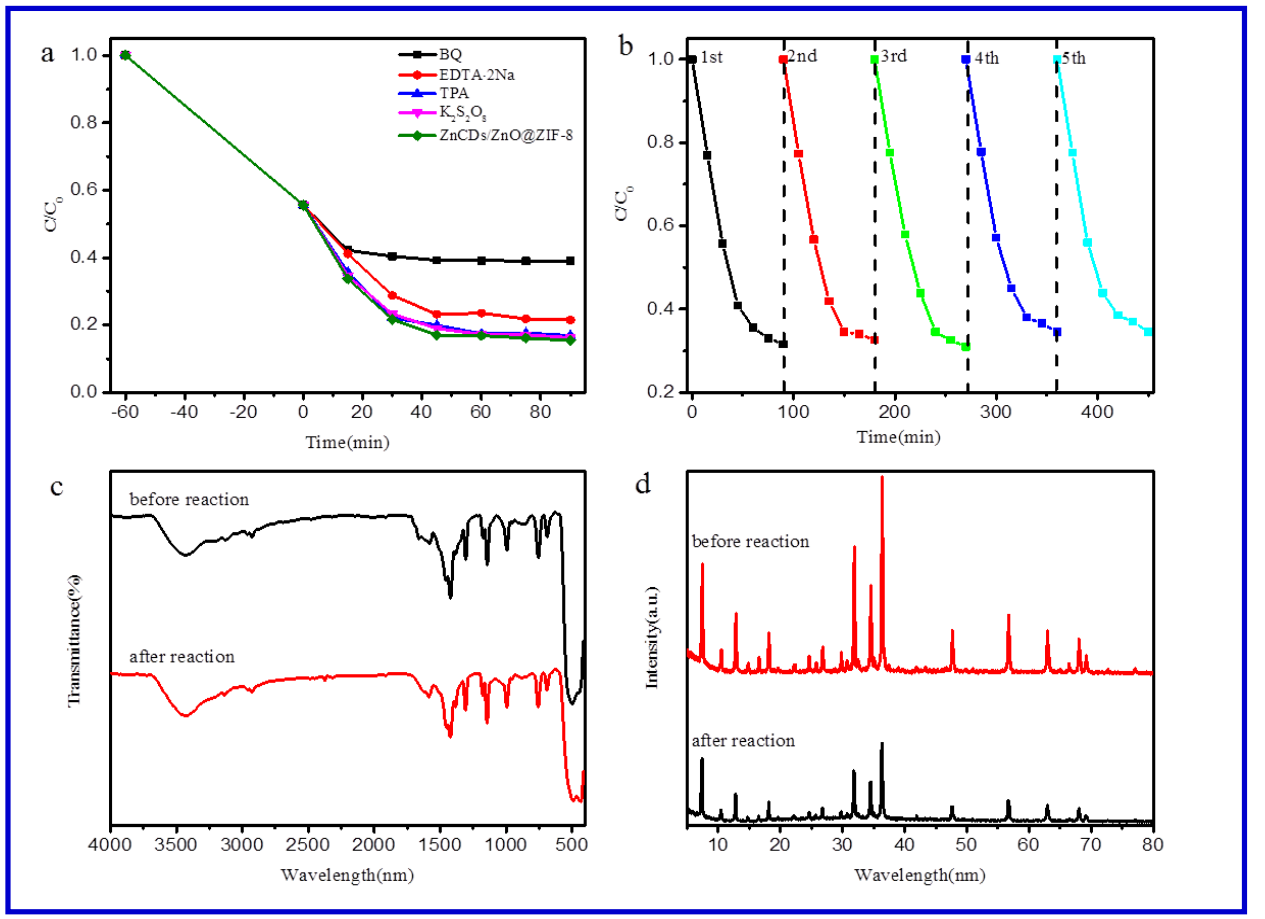

Figure 9. (a) Time-dependent photodegradation of TC over Zn-CDs/ZnO@ZIF-8 in the presence of different scavengers. (b) Cycling runs of Zn-CDs/ZnO@ZIF-8 (c) IR of Zn-CDs/ZnO@ZIF-8. (d) XRD of Zn-CDs/ZnO@ZIF-8 before and after reaction.

In order to ascertain the reactive species that directly participate in photocatalytic system, active species trapping experiments were tested. BQ, EDTA-2Na, $\mathrm{K}_{2} \mathrm{~S}_{2} \mathrm{O}_{8}$, and IPA were employed to quench $\cdot \mathrm{O}_{2-}, \mathrm{h}^{+}, \mathrm{e}^{-}$and $\cdot \mathrm{OH}$ in the photocatalytic process, respectively. As depicted in Figure 9a, the photocatalytic rate did not exhibit a noticeable change in the presence of EDTA-2Na, $\mathrm{K}_{2} \mathrm{~S}_{2} \mathrm{O}_{8}$, and IPA. This indicates that $\mathrm{h}^{+}, \mathrm{e}^{-}$, and $\cdot \mathrm{OH}$ that participated in the photocatalytic process did not play a leading role. Whereas, when $B Q$ was added to the reaction system, the degradation of TC was greatly inhibited. This result demonstrates that $\cdot \mathrm{O}_{2}{ }^{-}$is main reactive species for $\mathrm{ZnCDs} / \mathrm{ZnO} @ \mathrm{ZIF}-8$.

Furthermore, the stability of the photocatalyst was investigated through the cyclic degradation of TC under visible light irradiation. One hundred milligrams of $\mathrm{ZnCDs} / \mathrm{ZnO}$ @ZIF-8 was used in the recycling experiment. After the photocatalysis, the mixture was centrifuged to obtain a catalyst to characterize IR and XRD, or for the next reaction. It 
can be clearly seen that the photocatalytic activity remained almost constant after five cycles which proves the as-synthesized composites have high stability (Figure $9 b$ ). The incorporation of ZIF-8 into the $\mathrm{ZnCDs} / \mathrm{ZnO}$ nanospheres was therefore thought to enhance the visible light activity of $\mathrm{ZnO}$ and also inhibit the photo-corrosion of $\mathrm{ZnO}$, leading to a stable and durable photocatalytic activity. The IR spectra and XRD of ZnCDs/ZnO@ZIF-8 is almost unchanged before and after the photocatalytic reaction (Figure $9 c, d)$. It indicates that the catalyst is stable and recyclable during the photocatalytic process.

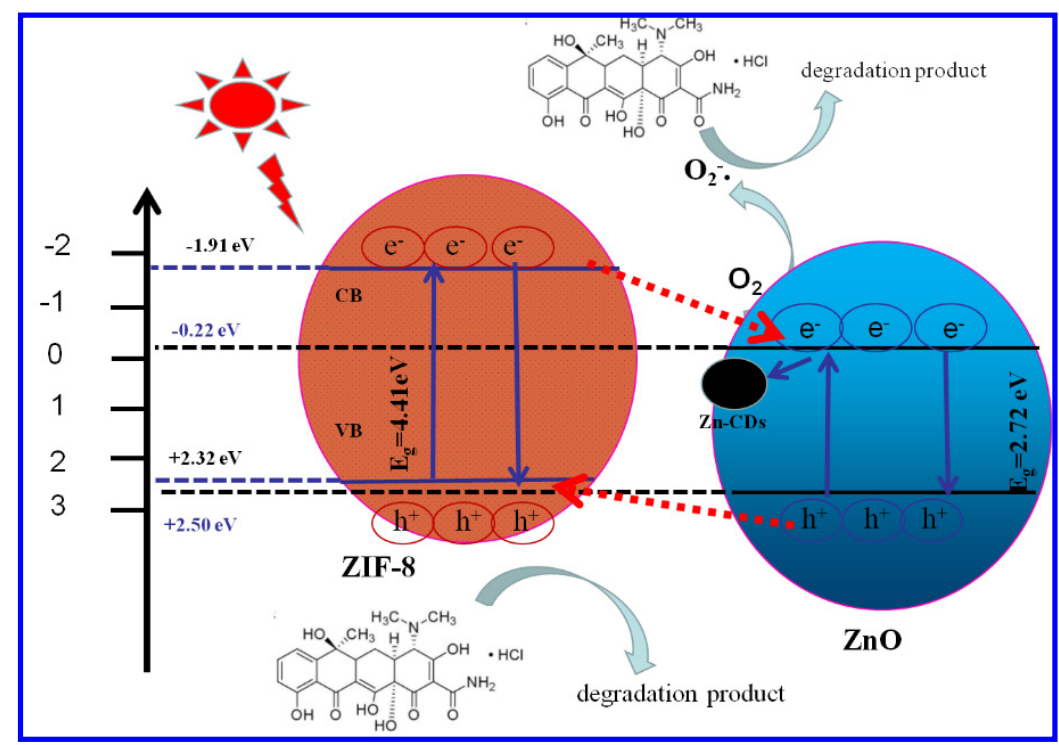

Figure 10. Proposed mechanism of photocatalytic degradation for TC.

On the basis of the above experimental results over ZnCDs/ZnO@ZIF-8, photocatalytic mechanism is proposed and illustrated in Figure 10. The photocatalytic activity of ZnCDs/ZnO@ZIF-8 can be attributed to the ZnCDs/ZnO heterojunction and ZIF-8, which effectively facilitates the electron transfer and the separation of electron-hole pairs. During UV irradiation, electrons and holes are excited at the conduction band (CB) and the valence band (VB) on the composites, respectively. After that, electrons transfer from the excited state to the $\mathrm{ZnCDs} / \mathrm{ZnO}$ and transform from $\mathrm{O}_{2}$ to $\cdot \mathrm{O}_{2-} \cdot \mathrm{O}_{2-}$ and holes at VB finally contribute to the degradation of tetracycline in the solution.

\section{Materials and Methods}

\subsection{Materials}

Zinc nitrate hexahydrate, DMF, alcohol were purchased from Sinopharm Group Chemical Reagent Co., Ltd. Shanghai, China. Tetracycline hydrochloride (TC) and 2-methyl imidazole (2-mIm) were purchase from Aladdin Chemical Reagent Co., Ltd. Shanghai, China. Folic acid was purchased from Macklin Technology Co., Ltd. Shanghai, China. Dialysis bag (MWCO: 1000 da) imported from the United States. All solvents and reagents were analytical grade and used without further purification.

\subsection{Characterization}

Fourier transform infrared spectroscopy (FT-IR) was recorded on a Nicolet iZ10 FT-IR Spectrophotometer (Thermo Electron Co., USA) with potassium bromide ( $\mathrm{KBr}$ ) as the dispersant to examine possible chemical bonds in adsorbents. X-ray diffraction (XRD) patterns were recorded on a German Brooke XRD-D8 Advance D8 with $\mathrm{Cu} \mathrm{K} \alpha$ radiation $(\lambda=0.15406 \mathrm{~nm}$, voltage $40 \mathrm{kV}$, current $40 \mathrm{~mA})$ and irradiated at a scan rate of $0.1^{\circ} / \mathrm{s}$ in the range of $5^{\circ}-80^{\circ}$. Ultraviolet-visible (UV-vis) spectra experiments were performed on Hitachi UV-vis 3010 spectrophotometer (Hitachi Ltd., Tokyo, Japan). The morphology and microstructure of the samples were characterized by scanning electron microscopy (SEM, 
Hitachi Ltd., Tokyo, Japan) and field emission scanning electron microscopy (FESEM, Hitachi S-4800, Hitachi Ltd., Tokyo, Japan) operated at $5 \mathrm{kV}$. X-ray photoelectron spectroscopy (XPS, Thermo Scientifific, EscaLab 250Xi spectrometer, USA) with a monochromatic Al $\mathrm{K} \alpha \mathrm{X}$-ray source was used to analyze elements $(\mathrm{hv}=1486.6 \mathrm{eV}$, power $150 \mathrm{~W}$, beam spot $650 \mathrm{um}$, voltage $14.8 \mathrm{kV}$, current $1.6 \mathrm{~A}$ and testing software is advantageous). Charge correction was carried out using polluted carbon $\mathrm{C} 1 \mathrm{~s}=284.8 \mathrm{eV}$. A constant analyzer pass energy $E_{p}$ narrow sweep $20 \mathrm{eV}$, wide sweep $100 \mathrm{eV}$, and vacuum degree $1 \times 10^{-10} \mathrm{MBA}$. Photoluminescence spectroscopy (PL) was carried out using a Hitachi F-4500 fluorescence spectrophotometer (Hitachi Ltd., Tokyo, Japan). The sample was excited by a $360 \mathrm{~nm}$ laser light source. UV-vis diffuse reflectance spectroscopy (DRS) were tested in absorption mode at the range of 200-800 $\mathrm{nm}$ by a Shimadzu UV-2450 spectrometer (Shimadzu Ltd., Tokyo, Japan) with $\mathrm{MgO}$ powder as the reference. The $\mathrm{MgO}$ powder was firstly pressed into thin disc on the holder, and then the powder sample was evenly spread on an $\mathrm{MgO}$ disc and pressed again. A $500 \mathrm{~W}$ Xenon lamp was used as the light source $(\lambda \geq 420 \mathrm{~nm})$. Electrochemical tests were carried out on a CHI760E electrochemical analyzer (CHIIns., Chenhua Ltd., Shanghai, China) in a conventional three-electrode configuration with a $\mathrm{Pt}$ foil as the counter electrode and $\mathrm{Ag} / \mathrm{AgCl}$ (saturated $\mathrm{KCl}$ ) as the reference electrode. The electrolyte solution was $1.0 \mathrm{~mol} \cdot \mathrm{L}^{-1} \mathrm{Na}_{2} \mathrm{SO}_{4}$. The working electrode was prepared by mixing $10 \mathrm{mg}$ catalyst with $10 \mu \mathrm{L} 5 \%$ Nafion and $10 \mathrm{~mL}$ ethyl alcohol to form a slurry, and then the slurry was coated onto a $1.0 \mathrm{~cm}^{2}$ ITO conductive glass. The scan rate was $0.1 \mathrm{~V} / \mathrm{sec}$ and the testing potential was $2 \mathrm{~V}$ to start the scan to sweep until $-2 \mathrm{~V}$, maintaining the scan window between $+2 \mathrm{~V}$ to $-2 \mathrm{~V}$ ( $\mathrm{vs} \mathrm{Ag} / \mathrm{AgCl}$ ) using $1 \mathrm{M} \mathrm{Na}_{2} \mathrm{SO}_{4}$ as supporting electrolyte. Specific surface areas of samples were measured using nitrogen sorption isotherms through a standard Brunauer-Emmett-Teller (BET) analysis (Tristar II 3020, Micromeritics Instrument Corporation, Norcross, GA, USA).

\subsection{Synthesis of $\mathrm{ZnCDs}$}

Folic acid $(0.1 \mathrm{~g})$ and DMF $(10 \mathrm{~mL})$ were mixed with $\mathrm{Zn}\left(\mathrm{NO}_{3}\right)_{2}(0.05 \mathrm{~g})$. The solution was stirred and then transferred into a $25 \mathrm{~mL}$ Telfon steel autoclave and heated at $190{ }^{\circ} \mathrm{C}$ for $12 \mathrm{~h}$. The solution of $\mathrm{ZnCDs}$ was obtained by dialysis against deionized water through a dialysis bag (MWCO: $1000 \mathrm{Da}$ ). It was evaporated to dryness for further use. A control sample without metal doping (bare CDs) was prepared under conditions similar to that of $\mathrm{ZnCDs}$.

\subsection{Synthesis of $\mathrm{ZnO}$}

Folic acid $(0.1 \mathrm{~g})$, DMF $(10 \mathrm{~mL})$ were mixed with $\mathrm{Zn}\left(\mathrm{NO}_{3}\right)_{2}(0.05 \mathrm{~g})$. The solution transferred into a $25 \mathrm{~mL}$ Telfon steel autoclave and heated at $190{ }^{\circ} \mathrm{C}$ for $12 \mathrm{~h}$. After the mixture was cooled down to room temperature, the precipitate was collected by centrifugation and washed with $\mathrm{H}_{2} \mathrm{O}$ and ethanol for several times. The solid was calcined at $500{ }^{\circ} \mathrm{C}$ for $4 \mathrm{~h}$ to obtain white powder $\mathrm{ZnO}$.

\subsection{Synthesis of $\mathrm{ZnCDs} / \mathrm{ZnO}$}

$\mathrm{ZnO}(100 \mathrm{mg})$ was mixed with $\mathrm{ZnCDs}(10 \mathrm{mg})$ in $\mathrm{CH}_{3} \mathrm{CH}_{2} \mathrm{OH}(20 \mathrm{~mL})$ and stirred for $6 \mathrm{~h}$. The precipitate obtained was washed with $\mathrm{H}_{2} \mathrm{O}$ and $\mathrm{CH}_{3} \mathrm{CH}_{2} \mathrm{OH}$, respectively, $\mathrm{ZnCDs} / \mathrm{ZnO}$ formed and then dried in vacuum.

\subsection{Synthesis of ZnCDs/ZnO@ZIF-8}

Twenty milliliters of methanolic solution including $\mathrm{ZnCDs} / \mathrm{ZnO}(80,100,200 \mathrm{mg})$, $160 \mathrm{mg}$ of zinc nitrate hexahydrate $(0.05 \mathrm{mmol})$, and $20 \mathrm{~mL}$ of methanolic solution including $410 \mathrm{mg}$ of 2-methylimidazole $(5 \mathrm{mmol})$ were mixed with stirred continuously at $50{ }^{\circ} \mathrm{C}$ for $4 \mathrm{~h}$. A white powder, ZnCDs/ZnO@ZIF-8, was collected by centrifugation, then washed with methanol and dried at $80^{\circ} \mathrm{C}$ for use. As a contrast, ZIF- 8 was prepared according to the procedure in the literature [47]. 


\subsection{Photocatalytic Degradation of Tetracycline}

The photocatalytic activity of ZnCDs/ZnO@ZIF-8 composites was determined by the photodegradation of tetracycline. The reaction mixture was irradiated by a $500 \mathrm{~W}$ Xenon lamp for photodegradation while being stirred. During the reaction, a cooling water circulation device was used to maintain the reaction temperature at around $50 \sim 60{ }^{\circ} \mathrm{C}$. In a typical process, $100 \mathrm{mg}$ of photocatalyst ZnCDs/ZnO@ZIF-8 was added in $1000 \mathrm{~mL}$ of the tetracycline solution and stirred for $60 \mathrm{~min}$ in the dark to attain anadsorption/desorption equilibration. For the normal concentration of TC present in the natural environment (in manure and soil, $0 \sim 200 \mathrm{mg} \cdot \mathrm{kg}^{-1}[48,49]$; in the aquatic environment, $0 \sim 20 \mu \mathrm{g} \cdot \mathrm{L}^{-1}[1,50]$ ); we chose $20 \mathrm{mg} \cdot \mathrm{L}^{-1}$ for the initial concentration of TC. In the photocatalytic process, $3 \mathrm{~mL}$ mixture was extracted and centrifuged to obtain clear liquor for the next residual TC detection using the spectrophotometer at a maximum absorption wavelength of $371 \mathrm{~nm}$. Photocatalytic efficiency was calculated by the following formula:

$$
\text { Photocatalytic efficiency }=\frac{\left(C_{0}-C\right)}{C_{0}} \times 100
$$

where $\mathrm{C}_{0}$ and $\mathrm{C}$ represent concentrations of tetracycline before and after irradiated, respectively.

Additives that could scavenge different active radicals were utilized in comparative trials to illuminate the degradation process of tetracycline. $\mathrm{K}_{2} \mathrm{~S}_{2} \mathrm{O}_{8}(1 \mathrm{mM})$, disodium ethylenediaminetetraacetic acid (EDTA-2Na, $1 \mathrm{mmol} \cdot \mathrm{L}^{-1}$ ), p-benzoquinone (BQ, $\left.1 \mathrm{mmol} \cdot \mathrm{L}^{-1}\right)$ and tert-butanol $\left(\mathrm{t}-\mathrm{BuOH}, 1 \mathrm{mmol} \cdot \mathrm{L}^{-1}\right)$ were introduced into the reaction system to capture electrons $\left(\mathrm{e}^{-}\right)$, holes $\left(\mathrm{h}^{+}\right)$, superoxide anion free radicals $\left(\cdot \mathrm{O}_{2}{ }^{-}\right)$, and hydroxyl free radicals $(\cdot \mathrm{OH})$. These tests were performed following the photocatalytic degradation process mentioned above.

\section{Conclusions}

In summary, we have prepared ZnCDs/ZnO@ZIF-8 composites via zeolite metalorganic frame ZIF-8 wrapped around $\mathrm{ZnCDs} / \mathrm{ZnO}$ heterojunction. The hybrid material exhibits superior photocatalysis performance for the degradation of tetracycline, which was induced by synergistic effect of $\mathrm{ZIF}-8$ and $\mathrm{ZnCDs} / \mathrm{ZnO}$. Our work may provide more thought to design zeolite MOF-decorated semiconductors and carbon dots-based composites that utilize visible light to solve the environmental problems.

Author Contributions: Y.C. designed the experiments and wrote them manuscript. C.J. supervised this study. X.W., D.W. and Y.M. supported the experiments and contributed to the scientific discussion. All authors have read and agreed to the published version of the manuscript.

Funding: This research was funded by the Natural Science Foundation of Education Department of Anhui Province. grant number: KJ2017A314.

Data Availability Statement: Data is contained within the article.

Acknowledgments: $Y$. Cheng is grateful to the Natural Science Foundation of Education Department of Anhui Province (KJ2017A314) for funding.

Conflicts of Interest: The authors declare no conflict of interest.

\section{References}

1. Bilal, M.; Mehmood, S.; Rasheed, T.; Iqbal, H.M.N. Antibiotics traces in the aquatic environment: Persistence and adverse environmental impact. Curr. Opin. Environ. Sci. Health. Health 2020, 13, 68-74. [CrossRef]

2. Cheng, D.; Ngo, H.H.; Guo, W.; Chang, S.W.; Nguyen, D.D.; Liu, Y.; Wei, Q.; Wei, D. A critical review on antibiotics and hormones in swine wastewater: Water pollution problems and control approaches. J. Hazard. Mater. 2020, 387, 121682. [CrossRef]

3. Välitalo, P.; Kruglova, A.; Mikola, A.; Vahala, R. Toxicological impacts of antibiotics on aquatic micro-organisms: A mini-review. Int. J. Hyg. Environ. Health 2017, 220, 558-569. [CrossRef]

4. Zhang, L.; Song, X.; Liu, X. Studies on the removal of tetracycline by multi-walled carbon nanotubes. Chem. Eng. J. 2011, 178, 26-33. [CrossRef] 
5. Wang, J.L.; Wang, S.Z. Preparation, modification and environmental application of biochar: A review. J. Clean. Prod. 2019, 227, 1002-1022. [CrossRef]

6. Wang, S.Z.; Hu, Y.M.; Wang, J.L. Biodegradation of typical pharmaceutical compounds by a novel strain Acinetobacter sp. J. Environ. Manag. 2018, 217, 240-246. [CrossRef] [PubMed]

7. Wu, J.; Zhang, H.; Oturan, N.; Wang, Y.; Chen, L.; Oturan, M.A. Application of response surface methodology to the removal of the antibiotic tetracycline by electrochemical process using carbon-felt cathode and $\mathrm{DSA}\left(\mathrm{Ti} / \mathrm{RuO}_{2}-\mathrm{IrO}_{2}\right)$ anode. Chemosphere 2012, 87, 614-620. [CrossRef]

8. Gao, Y.; Wu, J.; Wang, J.; Fan, Y.; Zhang, S.; Dai, W. A novel multifunctional p-type semiconductor@MOFs nanoporous platform for simultaneous sensing and photodegradation of tetracycline. ACS Appl. Mater. Interfaces 2020, 12, 11036-11044. [CrossRef] [PubMed]

9. Li, X.; Xiong, J.; Gao, X.; Ma, J.; Chen, Z.; Kang, B.; Liu, J.; Li, H.; Feng, Z.; Huang, J. Novel BP/BiOBr S-scheme nano-heterojunction for enhanced visible-light photocatalytic tetracycline removal and oxygen evolution activity. J. Hazard. Mater. 2020, 387, 121690. [CrossRef] [PubMed]

10. Zha, Z.; Lai, J.; Li, Y.; Yang, J.; Cui, S.; Li, Y. The degradation of tetracycline by modified BiOCl nanosheets with carbon dots from the chlorella. J. Alloys Compd. 2021, 855, 157454. [CrossRef]

11. Ponraj, Y.K.; Borah, B. Separation of methane from ethane and propane by selective adsorption and diffusion in MOF Cu-BTC: A molecular simulation study. J. Mol. Graph. Modell. 2020, 97, 107574. [CrossRef] [PubMed]

12. Wu, T.; Liu, X.J.; Liu, Y.; Cheng, M.; Liu, Z.F.; Zeng, G.M.; Shao, B.B.; Liang, Q.H.; Zhang, W.; He, Q.Y.; et al. Application of QD-MOF composites for photocatalysis: Energy production and environmental remediation. Coord. Chem. Rev. 2020, 403, 213097. [CrossRef]

13. Zhao, H.; Xing, Z.; Su, S.; Song, S.; Xu, T.; Li, Z.; Zhou, W. Recent advances in metal organic frame photocatalysts for environment and energy applications. Appl. Mater. Today 2020, 21, 100821. [CrossRef]

14. Zhang, J.; Wang, X.-Y.; Wang, Y.-H.; Wang, D.-D.; Song, Z.; Zhang, C.-D.; Wang, H.-S. Colorable zeolitic imidazolate frameworks for colorimetric detection of biomolecules. Anal. Chem. 2020, 92, 12670-12677. [CrossRef] [PubMed]

15. Huang, X.C.; Lin, Y.Y.; Zhang, J.P.; Chen, X.M. Ligand-directed strategy for zeolite-type metal-organic frameworks: Zinc(II) imidazolates with unusual zeolitic topologies. Angew. Chem. Int. Ed. 2006, 45, 1557-1559. [CrossRef]

16. Weber, M.D.R.; Baker, T.L.; Dao, B.; Kwon, C.; Tian, F.Y. Exploring the aggregative growth of nanoporous zeolitic imidazolate framework ZIF-8. Cryst. Growth Des. 2020, 20, 2305-2312. [CrossRef]

17. Wolf, A.; Diestel, L.; Lubkemann, F.; Kodanek, T.; Mohamed, T.; Caro, J.; Dorfs, D. Plasmonic semiconductor nanoparticles in a metal-organic framework structure and their in situ cation exchange. Chem. Mater. 2016, 28, 7511-7518. [CrossRef]

18. Jian, M.P.; Wang, H.; Liu, R.P.; Qu, J.H.; Wang, H.T.; Zhang, X.W. Self-assembled one-dimensional $\mathrm{MnO}_{2} @$ zeolitic imidazolate framework-8 nanostructures for highly efficient arsenite removal. Environ. Sci. Nano 2016, 3, 1186-1194. [CrossRef]

19. Zhu, K.; Chen, C.; Xu, H.; Gao, Y.; Tan, X.; Alsaedi, A.; Hayat, T. Cr(VI) reduction and immobilization by core-double-shell structured magnetic polydopamine@zeolitic idazolate frameworks-8 microspheres. ACS Sustain. Chem. Eng. 2017, 5, 6795-6802. [CrossRef]

20. Esken, D.; Turner, S.; Wiktor, C.; Kalidindi, S.B.; Van Tendeloo, G.; Fischer, R.A. GaN@ZIF-8: Selective formation of gallium nitride quantum dots. inside a zinc methylimidazolate framework. J. Am. Chem. Soc. 2011, 133, 16370-16373. [CrossRef]

21. Wang, X.B.; Liu, J.; Leong, S.; Lin, X.C.; Wei, J.; Kong, B.; Xu, Y.F.; Low, Z.-X.; Yao, J.F.; Wang, H.T. Rapid construction of ZnO@ZIF-8 heterostructures with size-selective photocatalysis properties. ACS Appl. Mater. Interface 2016, 8, 9080-9087. [CrossRef] [PubMed]

22. Yu, B.; Wang, F.; Dong, W.; Hou, J.; Lu, P.; Gong, J. Self-template synthesis of core-shell ZnO@ZIF-8 nanospheres and the photocatalysis under UV irradiation. Mater. Lett. 2015, 156, 50-53. [CrossRef]

23. Zhan, W.-W.; Kuang, Q.; Zhou, J.-Z.; Kong, X.-J.; Xie, Z.-X.; Zheng, L.-S. Semiconductor@metal-organic framework core-shell heterostructures: A case of ZnO@ZIF-8 nanorods with selective photoelectrochemical response. J. Am. Chem. Soc. 2013, 135, 1926-1933. [CrossRef]

24. Han, M.; Zhu, S.J.; Lu, S.Y.; Song, Y.B.; Feng, T.L.; Tao, S.Y.; Liu, J.J.; Yang, B. Recent progress on the photocatalysis of carbon dots: Classification, mechanism and applications. Nano Today 2018, 19, 201-218. [CrossRef]

25. Chu, K.-W.; Lee, S.L.; Chang, C.-J.; Liu, L.Y. Recent progress of carbon dot precursors and photocatalysis applications. Polymers 2019, 11, 689. [CrossRef]

26. Phang, S.J.; Tan, L.-L. Recent advances in carbon quantum dot (CQD)-based two dimensional materials for photocatalytic applications. Catal. Sci. Technol. 2019, 9, 5882-5905. [CrossRef]

27. Hazarika, D.; Karak, N. Photocatalytic degradation of organic contaminants under solar light using carbon dot/titanium dioxide nanohybrid, obtained through a facile approach. Appl. Surf. Sci. 2016, 376, 276-285. [CrossRef]

28. Zhao, W.; Yan, L.; Gu, H.; Li, Z.; Wang, Y.; Luo, Q.; Yang, Y.; Liu, X.; Wang, H.; Ma, C.-Q. Zinc oxide coated carbon dot nanoparticles as electron transport layer for inverted polymer solar cells. ACS Appl. Energy Mater. 2020, 3, 11388-11397. [CrossRef]

29. Zhang, X.-Y.; Liu, J.-K.; Wang, J.-D.; Yang, X.-H. Mass production, enhanced visible light photocatalytic efficiency, and application of modified $\mathrm{ZnO}$ nanocrystals by carbon dots. Ind. Eng. Chem. Res. 2015, 54, 1766-1772. [CrossRef]

30. Chin, M.; Cisneros, C.; Araiza, S.M.; Vargas, K.M.; Ishihara, K.M.; Tian, F. Rhodamine B degradation by nanosized zeolitic imidazolate framework-8 (ZIF-8). RSC Adv. 2018, 8, 26987-26997. [CrossRef] [PubMed] 
31. Wang, Y.; Ge, S.; Cheng, W.; Hu, Z.; Shao, Q.; Wang, X.; Lin, J.; Dong, M.; Wang, J.; Guo, Z. Microwave hydrothermally synthesized metal-organic framework-5 derived C-doped $\mathrm{ZnO}$ with enhanced photocatalytic degradation of Rhodamine B. Langmuir 2020, 36, 9658-9667. [CrossRef]

32. Lin, L.; Zhang, T.; Liu, H.; Qiu, J.; Zhang, X. In situ fabrication of a perfect Pd/ZnO@ZIF-8 core-shell microsphere as an efficient catalyst by a ZnO support-induced ZIF-8 growth strategy. Nanoscale 2015, 7, 7615-7623. [CrossRef]

33. Hu, Y.; Kazemian, H.; Rohani, S.; Huang, Y.; Song, Y. In situ high pressure study of ZIF-8 by FTIR spectroscopy. Chem. Commun. 2011, 47, 12694-12696. [CrossRef] [PubMed]

34. Zhao, S.; Lan, M.; Zhu, X.; Xue, H.; Ng, T.; Meng, T.; Lee, C.; Wang, P.; Zhang, W. Green synthesis of bifunctional fluorescent carbon dots from garlic for cellular imaging and free radical scavenging. ACS Appl. Mater. Interfaces 2015, 7, 17054-17060. [CrossRef]

35. Xiao, J.; Zhang, X.; Li, Y. A Ternary g- $\mathrm{C}_{3} \mathrm{~N}_{4} / \mathrm{Pt} / \mathrm{ZnO}$ photoanode for efficient photoelectrochemical water splitting. Int. J. Hydrogen Energy 2015, 40, 9080-9087. [CrossRef]

36. Mahala, C.; Sharma, M.D.; Basu, M. Type-II heterostructure of ZnO and carbon dots demonstrates enhanced photoanodic performance in photoelectrochemical water splitting. Inorg. Chem. 2020, 59, 6988-6999. [CrossRef]

37. Mahala, C.; Sharma, M.D.; Basu, M. Near-field and far-field plasmonic effects of gold nanoparticles decorated on ZnO nanosheets for enhanced solar water splitting. ACS Appl. Nano Mater. 2020, 3, 1153-1165. [CrossRef]

38. Biesingera, M.C.; Lau, L.W.M.; Gerson, A.R.; Smart, R.S.C. Resolving surface chemical states in XPS analysis of first row transition metals, oxides and hydroxides: Sc, Ti, V., Cu and Zn. Appl. Surf. Sci. 2010, 257, 887-898. [CrossRef]

39. Kruk, M.; Jaroniec, M. Gas adsorption characterization of ordered organic-inorganic nanocomposite materials. Chem. Mater. 2001, 13, 3169-3183. [CrossRef]

40. Gelb, L.D.; Gubbins, K.E. Pore size distributions in porous glasses: A computer simulation study. Langmuir 1999, 15, 305-308. [CrossRef]

41. Cheng, Y.; Mei, Y.; Deng, S.Y.; Li, J. In situ synthesis and photocatalytic performance of three dimensional composites CdS@DMSAGO. Chin. J. Inorg. Chem. 2020, 36, 714-729.

42. Li, Y.; Jiang, Y.; Ruan, Z.; Lin, K.; Yu, Z.; Zheng, Z.; Xu, X.; Yuan, Y. Simulation-guided synthesis of graphitic carbon nitride beads with $3 \mathrm{D}$ interconnected and continuous meso/macropore channels for enhanced light absorption and photocatalytic performance. J. Mater. Chem. A 2017, 5, 21300-21312. [CrossRef]

43. Bian, S.; Zhou, C.; Li, P.; Liu, J.; Dong, X.; Xi, F. Graphene quantum dots decorated titania nanosheets heterojunction efficient charge separation and enhanced visible-light photocatalytic performance. ChemCatChem 2017, 9, 3349-3357. [CrossRef]

44. Guo, F.; Shi, W.; Wang, H.B.; Han, M.; Guan, W.; Huang, H.; Liu, Y.; Kang, Z. Study on highly enhanced photocatalytic tetracycline degradation of type IIAgI/CuBi $\mathrm{O}_{4}$ and Z-scheme $\mathrm{AgBr} / \mathrm{CuBi}_{2} \mathrm{O}_{4}$ heterojunction photocatalysts. J. Hazard. Mater. 2018, 349, 111-118. [CrossRef]

45. Huang, W.Y.; Liu, N.; Zhang, X.D.; Wu, M.H.; Tang, L. Metal organic framework g- $\mathrm{C}_{3} \mathrm{~N}_{4} /$ MIL-53(Fe) heterojunctions with enhanced photocatalytic activity for Cr(VI) reduction under visible light. Appl. Surf. Sci. 2017, 425, 107-116. [CrossRef]

46. Yuan, D.; Ding, J.; Zhou, J.; Wang, L.; Wan, H.; Dai, W.-L.; Guan, G. Graphite carbon nitride nanosheets decorated with ZIF-8 nanoparticles: Effects of the preparation method and their special hybrid structures on the photocatalytic performance. J. Alloys Compd. 2018, 762, 98-108. [CrossRef]

47. Liang, X.; Quan, B.; Ji, G.; Liu, W.; Zhao, H.; Dai, S.; Lv, J.; Du, Y. Tunable dielectric performance derived from the metal-organic framework/reduced graphene oxide hybrid with broadband absorption. ACS Sustain. Chem. Eng. 2017, 5, 10570-10579. [CrossRef]

48. Hamscher, G.; Sczesny, S.; Hoper, H.; Nau, H. Determination of persistent tetracycline residues in soil fertilized with liquid manure by high-performance liquid chromatography with electrospray ionization tandem mass spectrometry. Anal. Chem. 2002, 74, 1509-1518. [CrossRef] [PubMed]

49. Ji, X.; Shen, Q.; Liu, F.; Ma, J.; Xu, G.; Wang, Y.; Wu, M. Antibiotic resistance gene abundances associated with antibiotics and heavy metals in animal manures and agricultural soils adjacent to feedlots in Shanghai, China. J. Hazard. Mater. 2012, 235, 178-185. [CrossRef]

50. Xu, L.; Zhang, H.; Xiong, P.; Zhu, Q.; Liao, C.; Jiang, G. Occurrence, fate, and risk assessment of typical tetracycline antibiotics in the aquatic environment: A review. Sci. Total Environ. 2021, 753, 141975. [CrossRef] 